\title{
PRODUCCIÓN CIENTÍFICA Y LICENCIAMIENTO DE ESCUELAS DE MEDICINA EN EL PERÚ
}

\author{
Percy Mayta-Tristán ${ }^{1, a}$, Carlos J. Toro-Huamanchumo²,b, Joel Alhuay-Quispe ${ }^{3, c}$, Josmel Pacheco-Mendoza ${ }^{3, d}$
}

\begin{abstract}
RESUMEN
La nueva ley universitaria 30220 del 2014 creó la obligatoriedad del licenciamiento institucional de todas las universidades peruanas a cargo de la Superintendencia Nacional de Educación Superior Universitaria - SUNEDU, el primer programa de pregrado en pasar por el mismo proceso será medicina. El licenciamiento de programas de medicina es necesario para garantizar que las condiciones en que se imparte la carrera en el Perú son las adecuadas, con la alta posibilidad de cierre de algunas escuelas de medicina. Una vez que una escuela de medicina haya demostrado que cumple con las condiciones básicas de calidad, se realiza una evaluación cualitativa y una cuantitativa que incluye tres criterios: producción científica en Web of Science, impacto medido a través del índice $\mathrm{H}$, y resultados del examen nacional de medicina, para determinar los años de licenciamiento. Este artículo realiza una evaluación de los indicadores cuantitativos vinculados a investigación usando Web of Science y Scopus, además de hacer revisiones técnicas y metodológicas de los mismos; así como sugerencias para los otros indicadores.
\end{abstract}

Palabras clave: Publicaciones; Investigación; Facultades de medicina; Educación médica; Acreditación; Bibliometría (fuente: DeCS BIREME).

\section{SCIENTIFIC PRODUCTION AND LICENSING OF MEDICAL SCHOOLS IN PERU}

\begin{abstract}
The new university law 30220 of 2014 introduced the mandatory institutional licensing of all Peruvian universities by the National Superintendence of Higher University Education (SUNEDU, in Spanish). The first undergraduate program to go through this process will be medicine. The licensing of medical programs is necessary to ensure that the conditions in which the program is taught in Peru are adequate, with a high probability of closing some medical schools. Once a medical school has demonstrated that it meets the basic conditions of quality, a qualitative and quantitative evaluation is carried out that includes three criteria: scientific production in the Web of Science, impact measured through the $\mathrm{H}$ index, and results of the national medical exam, to determine the years of licensing. This article evaluates the quantitative indicators linked to research using Web of Science and Scopus, in addition to making technical and methodological revisions of them. Suggestions for the other indicators are also covered by this article.
\end{abstract}

Keywords: Publications; Research; Medical Schools; Medical Education; Accreditation; Bibliometrics (source: MeSH NLM).

\section{INTRODUCCIÓN}

La investigación científica es uno de los ejes de la universidad peruana según la Ley Universitaria $\mathrm{N}^{\circ}$ $30220^{(1)}$, particularmente lo es en las escuelas de medicina donde se requiere del conocimiento del método científico para un abordaje adecuado del proceso de diagnóstico tratamiento de un paciente, así como para el desarrollo de competencias generales como la autonomía del aprendizaje, gestión de información, pensamiento crítico, comunicación y razonamiento cuantitativo ${ }^{(2-4)}$. Sin embargo, el proceso de enseñanza de investigación en las escuelas de medicina peruanas tiene ciertas falencias como bajo creditaje ${ }^{(5)}$, profesores, asesores y autoridades de investigación que no han publicado en revistas científicas ${ }^{(6-9)}$, cursos que no conducen al diseño, ejecución y comunicación adecuada de

\footnotetext{
Dirección General de Investigación, Desarrollo e Innovación, Universidad Científica del Sur. Lima, Perú.

Unidad de Investigación para la Generación y Síntesis de Evidencias en Salud, Universidad San Ignacio de Loyola. Lima, Perú.

Unidad de Investigación en Bibliometría, Universidad San Ignacio de Loyola. Lima, Perú.

a Médico salubrista, ${ }^{\mathrm{b}}$ Médico epidemiólogo, ${ }^{\mathrm{c}}$ Bibliotecólogo, ${ }^{\mathrm{d}}$ Veterinario, magister en gestión de información y del conocimiento.

Recibido: 21/02/2019 Aprobado: 06/03/2019 En línea: 20/03/2019
}

Citar como: Mayta-Tristán P, Toro-Huamanchumo CJ, Alhuay-Quispe J, Pacheco-Mendoza J. Producción científica y licenciamiento de escuelas de medicina en el Perú. Rev Peru Med Exp Salud Publica. 2019;36(1):106-15. doi:10.17843/rpmesp.2019.361.4315. 
la investigación ${ }^{(4,10-12)}$. Problemas con los asesores, tiempos y reglas no claras del proceso de tesis ${ }^{(13,14)}$, presencia de plagio ${ }^{(12,15)}$, así como limitado apoyo financiero para la investigación en pregrado ${ }^{(16)}$.

A pesar de estos problemas en el proceso de enseñanzaaprendizaje de la investigación en escuelas de medicina, el sector salud representa en promedio el $40 \%$ de la producción científica peruana en Scopus para el periodo 2000-2018 (Figura 1). En este periodo el Perú ha pasado de publicar 314 artículos en el 2000 a más de 3290 para el 2018, valor que ha duplicado lo que se publicó el 2013 (1566), se puede apreciar en la figura 1 que hay una inflexión en el crecimiento de las publicaciones peruanas a partir del 2014, fecha en que se aprobó la nueva ley universitaria $^{(1)}$. Si bien a nivel sudamericano Perú es el país que más ha crecido en producción científica en medicina en los últimos 20 años ${ }^{(17)}$, se evidencia que, en los últimos años la producción científica peruana ha incrementado a expensas de un crecimiento mayor en otros campos de la ciencia, disminuyendo el aporte de las ciencias de la salud de un $45 \%$ el 2015 al $36 \%$ el 2018 . Esto podría ser atribuido a un cambio en las políticas para estimular la investigación a partir de la ley universitaria, donde las áreas de conocimiento con menor costumbre de publicar en revistas indizadas han sido más susceptibles de mejora, por ejemplo, las ciencias sociales y humanidades pasaron de 330 artículos (15\%) el 2015 a 772 (23\%) el 2018.

Esta nueva ley, define que «la universidad es una comunidad académica orientada a la investigación y a la docencia» y ratifica a la investigación como uno de los ejes del modelo de toda universidad, y creó a la Superintendencia, creó a la Superintendencia Nacional de Educación Superior Universitaria (SUNEDU) que tiene como una de sus primeras actividades el evaluar a las 145 universidades peruanas vigentes al 2014, y según ello otorgar o denegar la licencia de funcionamiento. Para ello evalúa ocho condiciones básicas de calidad (CBC) que toda universidad peruana debe cumplir (Tabla 1).

La cuarta CBC es referida a las líneas de investigación y cuenta a su vez con ocho indicadores vinculados a investigación. Por ejemplo, que toda universidad tenga un Vicerrectorado de Investigación u órgano de gobierno similar, que cuente con líneas de investigación aprobadas, presupuesto, equipamiento, personal dedicado a investigación, registro de proyectos de investigación, código de ética, protección de propiedad intelectual, docentes registrados en el Directorio Nacional de Investigadores e Innovadores del Concytec (https://dina.concytec.gob.pe).

Adicionalmente, la evaluación de la producción científica (cantidad e impacto) de la universidad en Scopus, así como una evaluación cualitativa de la fortaleza de la política de investigación y carrera del investigador son consideradas para la determinación del tiempo de licencia institucional ${ }^{(18)}$. Esto ha significado que en los últimos años las universidades que tradicionalmente hacen investigación se consoliden y los investigadores sean más valorados, por otro lado, las otras universidades se han puesto a trabajar más seriamente en investigación, han implementado además de los vicerrectorados de investigación ${ }^{(19)}$, políticas de captación/retención de investigadores, estímulos y fondos de investigación, y una mayor vinculación con la investigación formativa.

Hasta febrero de 2019 (https://www.sunedu.gob.pe/ avances-licenciamiento/), se le ha denegado la licencia de funcionamiento a seis universidades que deben cerrarse

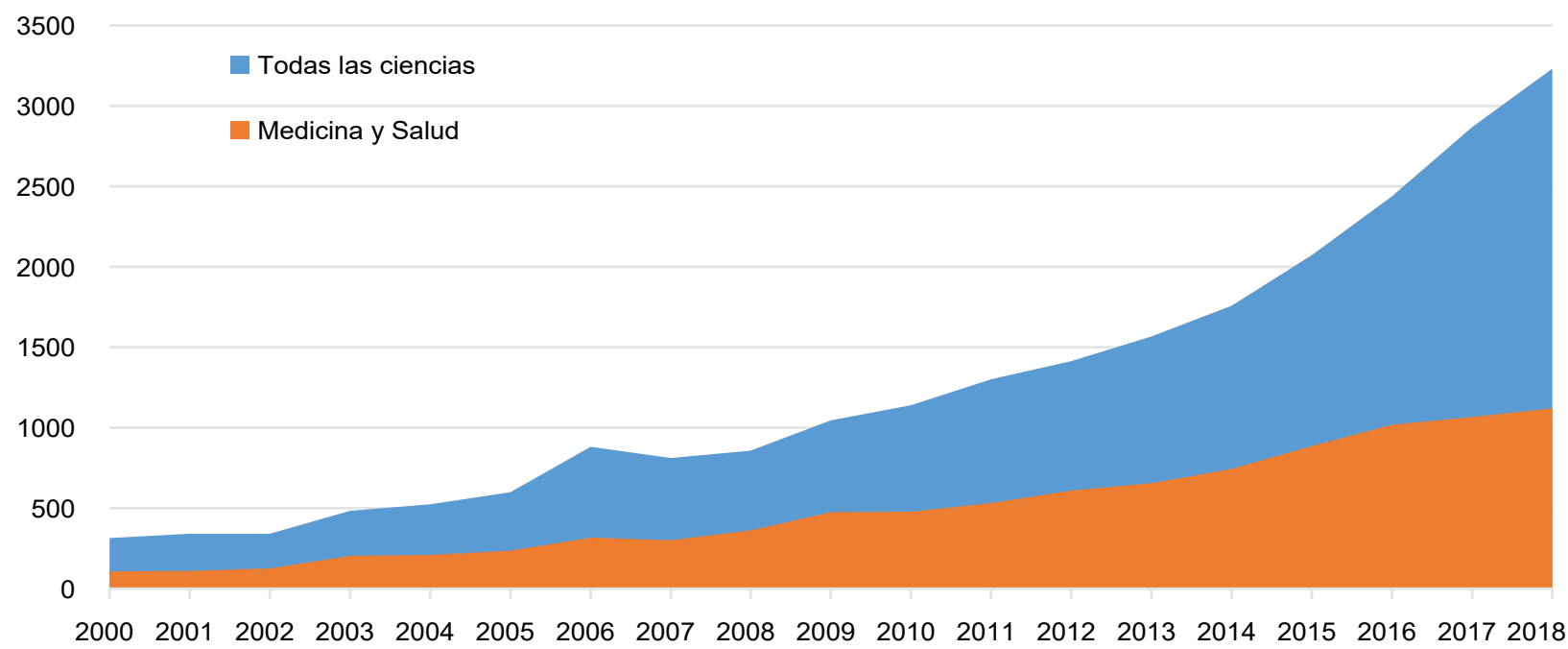

* Scopus el 13 de febrero de 2019. Los criterios para medicina y salud se tomaron de las áreas OCDE que SUNEDU usó para el informe bienal y considerará para el licenciamiento de programas de medicina.

Figura 1. Evolución de la producción científica peruana y en el área de medicina y salud en Scopus, 2000-2018* 
Tabla 1. Condiciones básicas de calidad (CBC) según el modelo de licenciamiento institucional de la Superintendencia Nacional de Educación Superior Universitaria (SUNEDU) ${ }^{(18)}$.

\begin{tabular}{lc}
\hline Condiciones & Indicadores \\
\hline CBC I. Existencia de objetivos académicos, grados y títulos a otorgar, y planes de estudios correspondientes & 8 \\
\hline CBC II. Oferta educativa a crearse compatible con los fines propuestos en los instrumentos de planeamiento & 7 \\
\hline $\begin{array}{l}\text { CBC III. Infraestructura y equipamiento adecuado al cumplimiento de sus funciones (aulas, bibliotecas, } \\
\text { laboratorios, entre otros). }\end{array}$ & 15 \\
\hline CBC IV. Líneas de investigación a ser desarrolladas. & 8 \\
$\begin{array}{l}\text { CBC V. Verificación de la disponibilidad de personal docente calificado con no menos de 25\% de docentes } \\
\text { a tiempo completo }\end{array}$ & 4 \\
$\begin{array}{l}\text { CBC VI. Verificación de los servicios educacionales complementarios básicos (servicio médico, social, } \\
\text { psicopedagógico, deportivo, entre otros) }\end{array}$ & 8 \\
\hline CBC VII. Existencia de mecanismos de mediación e inserción laboral (bolsa de trabajo u otros) & 4 \\
\hline CBC VIII. Transparencia de universidades & 1 \\
\hline Total & 55 \\
\hline
\end{tabular}

en un plazo de dos años, 60 universidades y dos escuelas de posgrado ya han sido licenciadas, y 77 universidades están aún en proceso de evaluación. Se espera que el 2019 todas las universidades hayan terminado el proceso de licenciamiento.

Luego de que SUNEDU determina que la universidad evaluada cumple con todas las condiciones básicas de calidad, el Consejo Directivo de SUNEDU decide el tiempo de licenciamiento basado en una evaluación cuantitativa de la investigación y una evaluación cualitativa de la carrera docente y de investigación ${ }^{(18)}$.

La evaluación cuantitativa se basa en el número de artículos e impacto normalizado en Scopus según el ranking Scimago 2015 (comprende el periodo 2009-2013 ${ }^{(20)}$ ) considerando los quintiles de distribución. Aquellas universidades que tengan en ambos el quintil superior les corresponde 10 años de licenciamiento, las que tuvieran al menos uno de los valores en el quintil superior y otro en el cuarto superior les corresponde 8 años, cualquier otra distribución le corresponde 6 años; sin embargo, este resultado está supeditado a la evaluación cualitativa basada en el informe del equipo de licenciamiento y la evaluación del Consejo Directivo de SUNEDU, como se puede apreciar en la Tabla 2.

Por ello, se han encontrado casos donde, correspondiéndole a la universidad un menor número de años por la evaluación cuantitativa (8 años), se le otorgó más años (10 años) debido a la fortaleza de su carrera docente y de investigación como es el caso de la Universidad Nacional Mayor de San Marcos y la Universidad Nacional de Ingeniería, en estos dos últimos casos cumplieron uno de los dos criterios cuantitativos. Sin embargo, a la Universidad del Pacífico le otorgaron más años sin cumplir ninguno de los criterios cuantitativos (Tabla 2).

\section{LICENCIAMIENTO DE ESCUELAS DE MEDICINA}

El proceso de licenciamiento incluye en una primera etapa el otorgamiento de la licencia a toda la universidad (licenciamiento institucional) y en una segunda etapa a los programas académicos de pregrado ${ }^{(1,18)}$. En ese contexto, el Comité de Educación Médica del Colegio Médico del Perú (21) solicitó a SUNEDU que sea medicina el primer programa a ser evaluado por el proceso de licenciamiento. En una editorial de Acta Médica Peruana ${ }^{(21)}$ se detectó que había 54 escuelas de medicina, cinco de ellas filiales, y tres con anuncio de funcionamiento. Con esa alerta SUNEDU detectó que tres escuelas de medicina estaban funcionando sin autorización por lo que fueron cerradas y las universidades, sancionadas (Universidad Autónoma del Perú, Universidad Privada Sergio Bernales y Universidad Arzobispo Loayza).

Es así que, en abril del 2017, la SUNEDU publicó un primer proyecto de la matriz de condiciones básicas de calidad y recibió el aporte de instituciones y ciudadanos. En diciembre de 2018 publicó el proyecto del modelo de licenciamiento ${ }^{(22)}$ y el reglamento de procedimiento ${ }^{(23)}$, dando hasta el 15 de enero de 2019 para recibir comentarios (https://www.sunedu. gob.pe/licenciamiento-programas-medicina-humana/). En ese contexto, varias instituciones y ciudadanos han enviado comentarios para mejorar su contenido, y es posible que varíen algunos de los indicadores, por lo que no vamos a profundizar en las condiciones básicas de calidad requeridas.

Uno de los aspectos incluidos es cómo se determinarán los años de licencia del programa de medicina, los cuales mantienen la lógica del licenciamiento institucional, pero con algunas diferencias, que pasaremos a revisar a continuación. 
Tabla 2. Universidades con ocho o más años de licenciamiento institucional basado en los criterios cuantitativos usando el Scimago Institution Rank 2015 (Scopus 2009-2013).

\begin{tabular}{|c|c|c|c|c|}
\hline \multirow[b]{2}{*}{ Universidad* } & \multicolumn{2}{|c|}{ Quintil Latinoamericano** } & \multirow{2}{*}{$\begin{array}{l}\text { Número de años } \\
\text { (evaluación } \\
\text { cuantitativa) }\end{array}$} & \multirow{2}{*}{$\begin{array}{l}\text { Número de años } \\
\text { otorgados }\end{array}$} \\
\hline & $\begin{array}{l}\text { Quintil (Número de } \\
\text { artículos) }{ }^{\dagger}\end{array}$ & $\begin{array}{l}\text { Quintil (Impacto } \\
\text { Normalizado) }{ }^{\ddagger}\end{array}$ & & \\
\hline Univ Peruana Cayetano Heredia & Quinto Sup (1228) & Quinto Sup $(1,18)$ & 10 & 10 \\
\hline Pontificia Univ Católica del Peru & Quinto Sup (644) & Quinto Sup $(1,44)$ & 10 & 10 \\
\hline Univ Nacional Mayor de San Marcos & Quinto Sup (892) & Cuarto Sup $(0,58)$ & 8 & 10 \\
\hline Univ Nacional Agraria La Molina & Cuarto Sup (188) & Quinto Sup $(1,15)$ & 8 & 8 \\
\hline Univ Nacional de Ingeniería & Cuarto Sup (153) & Quinto Sup $(0,83)$ & 8 & 10 \\
\hline Univ Nacional de San Antonio Abad & Cuarto Sup (116) & Quinto Sup $(1,87)$ & 8 & En evaluación \\
\hline Univ Nacional de la Amazonía Peruana & Cuarto Sup (70) & Quinto Sup $(1,43)$ & 8 & 8 \\
\hline Univ Nacional de Trujillo & Cuarto Sup (66) & Quinto Sup $(0,78)$ & 8 & 8 \\
\hline Univ de Piura & Cuarto Sup (57) & Quinto Sup $(0,92)$ & 8 & 8 \\
\hline Univ del Pacífico & Tercer Sup (40) & Segundo Sup $(0,31)$ & 6 & 8 \\
\hline \multicolumn{5}{|c|}{$\begin{array}{l}\text { * Las universidades no mencionadas no fueron incluidas al no cumplir los criterios para } 8 \text { o } 10 \text { años, o no les dieron } 8 \text { o } 10 \text { años de licencia institucional. } \\
\text { ** El quintil latinoamericano fue calculado por los autores en función de los reportes del ranking Scimago } 2015^{(20)} \text { replicando lo que mencionó SUNEDU } \\
\text { que realizó. Se ordenó a todas las universidades en función de los indicadores reportados y se obtuvo los quintiles respectivos. Se reporta el quintil y el } \\
\text { valor del indicador. } \\
\dagger \quad \text { Número de artículos publicados por la Universidad en Scopus en el periodo } 2009-2013\end{array}$} \\
\hline \multicolumn{5}{|c|}{$\begin{array}{l}\text { Fos valores (en números decimales) muestran la relación entre el impacto científico promedio de una institución y el promedio mundial fijado en una } \\
\text { puntuación de } 1 \text {. Un "impacto normalizado" de } 0,7 \text { significa que la institución es citada un } 30 \% \text { por debajo del promedio mundial, mientras que } 1,2 \\
\text { significa la institución es citada un } 20 \% \text { por encima del promedio. }\end{array}$} \\
\hline
\end{tabular}

\section{DETERMINACIÓN DE AÑOS DE LICENCIA DE PROGRAMAS DE MEDICINA}

El documento en consulta ${ }^{(22)}$ plantea tres indicadores cuantitativos y no menciona como se evaluará el aspecto cualitativo. Los detalles generales se abordan en la tabla 3. La propuesta de los criterios cuantitativos está basada en la producción científica y resultados de egresados, y ha sido adecuada a las características del programa de medicina, y si bien esta propuesta es adecuada a las características del programa de medicina, hay varios considerandos técnicos que deben ser conocidos para cualquier ajuste futuro a estos criterios, ya sea a nivel institucional, de medicina $u$ otros programas.

Medición de la producción científica. Para el licenciamiento institucional se usó la cantidad de artículos en Scopus medida por el grupo Scimago a través del ranking de universidades iberoamericanas ${ }^{(20)}$, este procedimiento tiene ciertas ventajas: a) La evaluación es realizada por una institución independiente y los datos son públicos y transparentes; b) Scimago desambigua las filiaciones de cada institución por lo que se tiene la mejor aproximación a la real producción de cada institución ${ }^{(24)}$; c) Scopus contiene mayor número de publicaciones peruanas que Web of Science (WoS) para el 2018 (3255 frente a 1129), mayor número de revistas peruanas indizadas (nueve frente a ninguna), y mayor número de universidades peruanas con identificador institucional (ID) que WoS InCites (78 frente a 51); d) Permite el cálculo de quintiles o percentiles a nivel de Latinoamérica, ello condiciona que las universidades peruanas se comparen con sus pares de la región, y tengan mayor estímulo para mejorar ${ }^{(25)}$. Por otro lado, varios sistemas de evaluación se prefiere el uso de WoS al ser considerada la base de datos más selectiva, además de ser la primera que midió el impacto de las publicaciones científicas.

Sin embargo, el ranking Scimago no se genera por campos del conocimiento ${ }^{(25)}$, por lo que no sería útil si se quiere evaluar la producción específica de una carrera. Tanto en WoS como en Scopus, la asignación al campo de conocimiento depende de la clasificación asignada a la revista, y no al artículo o a la filiación institucional de los autores ${ }^{(26,27)}$. Por tanto, la medición de un campo de conocimiento de una universidad en estas bases de datos no refleja en forma fidedigna la producción específica de un determinado departamento, escuela o facultad, aspecto que se pudo ver, por ejemplo, en el informe bienal de SUNEDU del $2018^{(28)}$.

Si bien a través de estrategias de búsquedas se puede medir la producción científica en el área de medicina y salud (Anexo 1 - visualizar en versión electrónica) en Web of Science usando InCites y Scopus, hay ciertas diferencias que hemos encontrado en el proceso: a) Con Scopus no podemos generar un ranking latinoamericano en forma manual y rápida por problemas en el proceso de extracción de datos; b) El número de universidades peruanas con escuelas de medicina recopiladas con InCites es menor que las que tiene Scopus con ID affiliation (31 frente a 44); c) En salud se prefiere publicar en Medline, y todo Medline es recogido por Scopus, por lo que la brecha 
Tabla 3. Criterios para la determinación de años de licencia institucional y de medicina por la SUNEDU.

\begin{tabular}{|c|c|c|c|}
\hline Criterios & $\underset{(18)}{\text { Licenciamiento Institucional }}$ & Licenciamiento Medicina ${ }^{(22)}$ & Comentarios de los autores \\
\hline \multicolumn{4}{|l|}{ Cuantitativo } \\
\hline $\begin{array}{l}\text { Producción } \\
\text { científica }\end{array}$ & $\begin{array}{c}\text { Número de artículos totales } \\
\text { publicados en Scopus y } \\
\text { recopilados por el Scimago } \\
\text { Institution Ranking - SIR (periodo } \\
5 \text { años), ordenados por quintiles } \\
\text { de Latinoamérica }\end{array}$ & $\begin{array}{l}\text { Número de artículos en medicina } \\
\text { y salud en Web of Science Core } \\
\text { Collection recopilados por InCites } \\
\text { (periodo } 5 \text { años, sin ESCl), ordenados } \\
\text { por quintiles de Latinoamérica }\end{array}$ & $\begin{array}{c}\text { No existe un ranking (como el SIR) por } \\
\text { campo de conocimiento, la construcción } \\
\text { de ranking latinoamericano sólo se puede } \\
\text { hacer a través de un trabajo manual o } \\
\text { sistemas de cosecha como Scival-Scopus } \\
\text { o InCites de WoS. }\end{array}$ \\
\hline $\begin{array}{l}\text { Impacto de } \\
\text { la producción } \\
\text { científica }\end{array}$ & $\begin{array}{l}\text { Impacto normalizado* de la } \\
\text { producción científica ordenados } \\
\text { por quintiles de Latinoamérica. }\end{array}$ & $\begin{array}{c}\text { Índice } \mathrm{H}^{\star *} \text { de la producción } \\
\text { científica ordenados por quintiles de } \\
\text { Latinoamérica }\end{array}$ & $\begin{array}{l}\text { El Índice } \mathrm{H} \text { en un periodo específico es } \\
\text { un indicador más estable que el impacto } \\
\text { normalizado para instituciones con poca } \\
\text { producción como las peruanas. }\end{array}$ \\
\hline $\begin{array}{l}\text { Calidad del } \\
\text { egresado }\end{array}$ & No incluido & $\begin{array}{c}\text { Resultados del Examen Nacional } \\
\text { de Medicina (ENAM) ordenados } \\
\text { por quintiles del país }\end{array}$ & $\begin{array}{c}\text { Ordenados por la mediana del ENAM } \\
\text { ordinario considerando a toda la cohorte } \\
\text { de egresados, hayan dado o no el ENAM } \\
\text { ordinario. }\end{array}$ \\
\hline \multicolumn{4}{|l|}{ Cualitativo } \\
\hline Carrera docente & $\begin{array}{l}\text { A partir de los criterios básicos } \\
\text { de calidad }\end{array}$ & No mencionado & $\begin{array}{l}\text { Debería tomarse en cuenta en forma } \\
\text { explícita la relación estudiante / docente } \\
\text { tiempo completo }\end{array}$ \\
\hline $\begin{array}{l}\text { Política de } \\
\text { investigación }\end{array}$ & $\begin{array}{l}\text { A partir de los criterios básicos } \\
\text { de calidad }\end{array}$ & No mencionado & $\begin{array}{l}\text { Debería tomarse en cuenta en forma } \\
\text { explícita el número de investigadores } \\
\text { reconocidos (REGINA o similar) y la } \\
\text { articulación de la investigación con la } \\
\text { formación de los estudiantes }\end{array}$ \\
\hline
\end{tabular}

* Relación entre el impacto científico promedio de una institución y el promedio mundial fijado en una puntuación de 1.

${ }^{* *}$ Equivale al dígito que equipara cuantitativamente las publicaciones de una institución y las citas que éstas han obtenido en el periodo y base de datos evaluada.

entre lo disponible en Scopus frente a WoS es mayor (5559 frente a 2972 artículos en salud en el periodo 2013-2018).

\begin{abstract}
Medición del impacto de la producción científica. Existen varios indicadores de impacto basados en citas ${ }^{(29-31)}$, para el licenciamiento institucional se usó el impacto normalizado, sin embargo, este dato es poco estable cuando el número de artículos es menor a $100{ }^{(25)}$, como sucede en la mayoría de universidades peruanas. Por ello, la decisión de usar el Índice $\mathrm{H}$ del periodo evaluado es acertada. Ya que se toma en cuenta tanto las citas como la producción total, se puede calcular tanto a nivel global como por campos de conocimiento, y no se generan distorsiones cuando la muestra (número de artículos) es pequeña ${ }^{(32,33)}$.
\end{abstract}

Estimación de los indicadores de producción científica y de impacto. Usando el mismo sistema de evaluación bibliométrica de WoS InCites excluyendo a las revistas ESCI (Emerging Source Citation Index), se construyó la relación de campos de conocimiento (Anexo 2 - visualizar en versión electrónica) para obtener sólo las publicaciones de medicina y salud en el periodo 2013-2018 para todas las instituciones académicas de los países de Latinoamérica, tal como plantea SUNEDU en su propuesta ${ }^{(22)}$. Después se extrajo los resultados de cantidad de artículos, índice $\mathrm{H}$ del periodo y los respectivos quintiles en función de las universidades latinoamericanas existentes (Tabla 4).

Dado que la evaluación de SUNEDU se basa en quintiles latinoamericanos se requiere construir un ranking latinoamericano de escuelas de medicina, idealmente deberíamos tener el listado de escuelas de medicina de todos los países de Latinoamérica y hacer el mismo procedimiento individual que podemos hacer para Perú porque conocemos esta información. Sin embargo, existen dificultades técnicas para ello.

Por ejemplo, hay que precisar que el registro de universidades latinoamericanas en WoS Incites es incompleto. Perú representa el 9,3\% (51/551) de instituciones en este reporte frente al 4,3\% (77/1771) en el Ranking Scimago de Universidades Iberoamericanas 2018. Esta subrepresentación puede afectar la distribución de los quintiles; sin embargo, dado los resultados de las instituciones (Tabla 4), sólo hay tres universidades que potencialmente pueden verse afectadas (la tercera, cuarta y quinta con mayor producción), ya que las dos primeras se mantendrían en el quintil superior, y la distancia entre la quinta y sexta institución es bastante amplia, por lo que no afectaría las posiciones para un cambio en la decisión de años de licenciamiento en las universidades con menor producción, al menos en el aspecto cuantitativo vinculado a investigación.

En la Tabla 4 presentamos también la cantidad de artículos e índice $\mathrm{H}$ en Scopus, las estrategias de búsqueda para conocer la producción científica de medicina y salud de cada universidad peruana con escuela de medicina puede apreciarse en el Anexo 1. Sin embargo, no nos fue factible 
Tabla 4. Producción científica en medicina y salud 2013-2018 y estimación del periodo de licencia de escuelas de medicina peruanas

\begin{tabular}{|c|c|c|c|c|c|c|c|c|}
\hline \multirow{2}{*}{ Universidades } & \multicolumn{3}{|c|}{$\begin{array}{c}\text { Publicaciones en Medicina y } \\
\text { Salud 2013-2018*t }\end{array}$} & \multicolumn{3}{|c|}{$\begin{array}{c}\text { Índice H en Medicina y } \\
\text { Salud 2013-2018*\$ }\end{array}$} & \multirow{2}{*}{$\begin{array}{l}\text { Licencia } \\
\text { Institucional ** } \\
\text { (años) }\end{array}$} & \multirow{2}{*}{$\begin{array}{c}\text { Periodo de } \\
\text { licencia máximo } \\
\text { de medicina }{ }^{* * *} \\
\text { (años) }\end{array}$} \\
\hline & $\begin{array}{c}\text { WoS } \\
\text { InCites }\end{array}$ & Scopus & $\begin{array}{l}\text { Quintil } \\
\text { Latam }\end{array}$ & $\begin{array}{c}\text { WoS } \\
\text { InCites }\end{array}$ & Scopus & $\begin{array}{l}\text { Quintil } \\
\text { Latam }\end{array}$ & & \\
\hline Univ Peruana Cayetano Heredia & 1665 & 1724 & Quinto & 44 & 48 & Quinto & 10 & 10 \\
\hline Univ Nac Mayor de San Marcos & 488 & 630 & Quinto & 19 & 22 & Quinto & 10 & 10 \\
\hline Univ Peruana de Ciencias Aplicadas & 324 & 541 & Cuarto & 17 & 20 & Cuarto & 6 & 8 \\
\hline Univ de San Martín de Porres & 152 & 310 & Cuarto & 15 & 15 & Cuarto & 6 & 8 \\
\hline Univ Científica del Sur & 111 & 128 & Tercer & 9 & 9 & Tercer & 6 & 6 \\
\hline Univ Ricardo Palma & 47 & 90 & Tercer & 5 & 7 & Segundo & 6 & 6 \\
\hline Univ Nac de San Antonio Abad & 29 & 56 & Segundo & 5 & 6 & Segundo & En evaluación & 6 \\
\hline Univ Nac de la Amazonia Peruana & 27 & 35 & Segundo & 6 & 8 & Tercer & 8 & 6 \\
\hline Univ Católica Santa María & 25 & 36 & Segundo & 3 & 4 & Segundo & 6 & 6 \\
\hline Univ Nac de San Agustín & 23 & 52 & Segundo & 4 & 5 & Segundo & 10 & 6 \\
\hline Univ Nac Federico Villarreal & 22 & 41 & Segundo & 3 & 5 & Segundo & En evaluación & 6 \\
\hline Univ Nac de Piura & 19 & 51 & Segundo & 3 & 5 & Segundo & En evaluación & 6 \\
\hline Univ Nac San Luis Gonzaga & 18 & 57 & Segundo & 1 & 4 & Primer & En evaluación & 6 \\
\hline Univ Privada San Juan Bautista & 17 & 26 & Segundo & 3 & 3 & Segundo & En evaluación & 6 \\
\hline Univ Privada Antenor Orrego & 17 & 85 & Segundo & 2 & 4 & Primer & 6 & 6 \\
\hline Univ Continental & 17 & 68 & Segundo & 2 & 5 & Primer & 6 & 6 \\
\hline Univ Nac Pedro Ruiz Gallo & 17 & 32 & Segundo & 2 & 3 & Primer & En evaluación & 6 \\
\hline Univ Nac del Altiplano & 13 & 19 & Segundo & 5 & 4 & Segundo & 6 & 6 \\
\hline Univ Nac de Cajamarca & 12 & 16 & Primer & 3 & 4 & Segundo & 6 & 6 \\
\hline Univ Nac de Trujillo & 10 & 63 & Primer & 2 & 4 & Primer & 8 & 6 \\
\hline Univ Andina del Cusco & 6 & 9 & Primer & 3 & 3 & Segundo & 6 & 6 \\
\hline Univ de Piura & 6 & 11 & Primer & 2 & 1 & Primer & 8 & 6 \\
\hline $\begin{array}{l}\text { Univ Nac de San Cristóbal de } \\
\text { Huamanga }\end{array}$ & 6 & 11 & Primer & 1 & 4 & Primer & 6 & 6 \\
\hline Univ Privada de Tacna & 5 & 10 & Primer & 1 & 2 & Primer & 6 & 6 \\
\hline Univ Alas Peruanas & 2 & 5 & Primer & 2 & 2 & Primer & En evaluación & 6 \\
\hline Univ Peruana Los Andes & 2 & 8 & Primer & 2 & 1 & Primer & En evaluación & 6 \\
\hline Univ Peruana Unión & 2 & 3 & Primer & 1 & 0 & Primer & 6 & 6 \\
\hline Univ Nac Hermilio Valdizán & 2 & 7 & Primer & 0 & 3 & Primer & En evaluación & 6 \\
\hline Univ Nac Jorge Basadre Grohmann & 1 & 3 & Primer & 1 & 1 & Primer & 6 & 6 \\
\hline Univ Privada Cesar Vallejo & 1 & 22 & Primer & 1 & 3 & Primer & En evaluación & 6 \\
\hline Univ Nac De Ucayali & 1 & 9 & Primer & 0 & 1 & Primer & 6 & 6 \\
\hline $\begin{array}{l}\text { Univ Católica Santo Toribio de } \\
\text { Mogrovejo }\end{array}$ & - & 29 & No aplica & - & 2 & No aplica & 6 & 6 \\
\hline Univ Señor de Sipán & - & 11 & No aplica & - & 1 & No aplica & En evaluación & 6 \\
\hline Univ Particular de Chiclayo & - & 9 & No aplica & - & 1 & No aplica & En evaluación & 6 \\
\hline Univ Nac del Centro del Perú & - & 8 & No aplica & - & 1 & No aplica & En evaluación & 6 \\
\hline $\begin{array}{l}\text { Univ Andina Néstor Cáceres } \\
\text { Velásquez }\end{array}$ & - & 6 & No aplica & - & 1 & No aplica & En evaluación & 6 \\
\hline Univ Privada Norbert Wiener & - & 7 & No aplica & - & 2 & No aplica & En evaluación & 6 \\
\hline $\begin{array}{l}\text { Univ Nac José Faustino Sánchez } \\
\text { Carrión }\end{array}$ & - & 5 & No aplica & - & 1 & No aplica & En evaluación & 6 \\
\hline Univ San Pedro**** & - & 3 & No aplica & - & 1 & No aplica & En evaluación & 6 \\
\hline Univ Nac del Santa & - & 2 & No aplica & - & 0 & No aplica & En evaluación & 6 \\
\hline Univ Nac Daniel Alcides Carrión & - & 4 & No aplica & - & 3 & No aplica & En evaluación & 6 \\
\hline Univ Nac de Tumbes & - & 3 & No aplica & - & 1 & No aplica & En evaluación & 6 \\
\hline $\begin{array}{l}\text { Univ Nac Toribio Rodríguez de } \\
\text { Mendoza }\end{array}$ & - & 0 & No aplica & - & 0 & No aplica & 6 & 6 \\
\hline Univ Nac de San Martín & - & 0 & No aplica & - & 0 & No aplica & En evaluación & 6 \\
\hline Univ Privada Telesup & - & - & No aplica & - & - & No aplica & En evaluación & 6 \\
\hline
\end{tabular}

* Medición realizada el 10 febrero de 2019.

† Número de artículos publicados por la Universidad en el periodo 2013-2018 en Scopus y WoS Incites.

$\ddagger$ Equivale al dígito que equipara cuantitativamente las publicaciones de una institución y las citas que éstas han obtenido en el periodo $2013-2018$ en la base de datos evaluada. Un índice $\mathrm{H}=3$ indica que la institución tiene al menos 3 artículos publicados que han recibido 3 citas o más cada uno de ellos en ese periodo y en esa base de datos.

** Verificado en las resoluciones de licenciamiento institucional de cada universidad, disponible en: https://www.sunedu.gob.pe/lista-de-universidades-licenciadas/

*** Considerando sólo los dos indicadores de producción científica, pendiente evaluar resultados del examen nacional de medicina y la evaluación cualitativa.

**** No tiene ID-AF en Scopus, pero se calculó a partir de búsqueda específica

No aplica: no cuenta con publicaciones recogidas con su identificador en esa base de datos y por tanto no se puede asignar en alguno de los quintiles. Latam: Latinoamérica 
encontrar una estrategia simple, rápida y replicable para calcular un ranking latinoamericano usando WoS sin InCites, Scopus o Scival. En el caso de WoS, porque que no hay ID públicos de cada institución, y en Scopus y SciVAL, si bien cuentan con ID públicos de instituciones, tienen limitaciones para la extracción de datos (sólo permite extraer hasta 160 ID).

En caso a futuro, se decida trabajar con percentiles sólo de universidades peruanas (decisión que recomendamos), Scopus sería una mejor alternativa que WoS, particularmente para carreras vinculadas a salud y ciencias de la vida, dado que tiene mayor número de artículos de autores peruanos, revistas peruanas indizadas y recopila todo Medline, que es la base de datos más usada y con más prestigio en el campo de las ciencias de la salud.

El Examen Nacional de Medicina. Es a la fecha la única herramienta disponible para evaluar las competencias de los egresados de escuelas de medicina en el Perú, es administrado por la Asociación Peruana de Facultades de Medicina (ASPEFAM) ${ }^{(34)}$, requisito necesario para hacer el SERUMS ${ }^{(35)}$. En algún momento se planteó la obligatoriedad de aprobarlo para hacer el SERUMS pero el Ministerio de Salud retrocedió ${ }^{(36)}$, y a fines de enero de 2019 el Colegio Médico del Perú ha decidido que será un requisito aprobarlo para que un médico pueda colegiarse ${ }^{(37)}$.

Este examen suele aplicarse al finalizar la carrera cuando el estudiante está terminando el internado (ENAM ordinario), y aquellos que no pudieron darlo, tienen la oportunidad de hacerlo meses después (ENAM extraordinario). Dado que algunas universidades plantearon como riesgo que la posición de una universidad, a través del promedio promocional, pueda usarse como estrategia comercial, decidieron que los resultados no sean públicos desde el año $2013^{(38)}$. Sin embargo, estos resultados han sido calculados y filtrados en los subsiguientes años, adicionalmente se han detectado malas prácticas en algunas escuelas para "incrementar" su promedio, como el que no todos los estudiantes rindan el ENAM ordinario, haciendo que estudiantes con menor rendimiento esperen al ENAM extraordinario. Esto podría distorsionar la forma de medir este indicador.

Por ello, se sugiere que para calcular los quintiles de distribución: (a) se consideren a todas las escuelas de medicina que hayan tenido egresados en el año de medición del ENAM para el licenciamiento, (b) sólo se incluya el ENAM ordinario, (c) que no se use el promedio sino la mediana considerando a toda la cohorte de egresados que debían rendir el ENAM, colocando cero a quienes no dieron el examen, dado que no se está usando el promedio este valor no afectaría la medida de tendencia central. Finalmente, recomendamos fuertemente que una universidad donde la mediana de sus egresados tiene nota desaprobatoria no obtenga el licenciamiento del programa, o que pase a plan de adecuación hasta demostrar que sus estudiantes mejoran su rendimiento y con un tiempo de licenciamiento menor ( 3 años) hasta demostrar una mejora sostenible en sus resultados.

Evaluación cualitativa. Si bien en la propuesta preliminar de SUNEDU ${ }^{(22)}$ no mencionó la evaluación cualitativa como en el licenciamiento institucional ${ }^{(18)}$, es muy probable que en la versión final se incluya, más aún cuando en algunos casos tuvo mayor peso que la evaluación cuantitativa. A pesar que el licenciamiento sólo evalúa condiciones básicas de calidad, la intención de la evaluación cualitativa es ponderar aquellos aspectos que pueden asegurar un mejor resultado en la formación de los estudiantes, como es la carrera docente y políticas de investigación sostenibles.

En ese contexto, hay indicadores que no son medidos como CBC pero que pueden ser más objetivos para la evaluación cualitativa como la relación de estudiantes sobre profesores a tiempo completo ${ }^{(39)}$, o el número de investigadores reconocidos por Concytec que son parte de la universidad y cómo está articulada la investigación que se realiza con la formación de estudiantes, de tal manera que permita al estudiante hacer investigación. Estos criterios posiblemente apliquen mucho más a un licenciamiento institucional (toda la universidad) que a uno de programas (p.e. medicina). Se espera que en la versión final del documento sobre evaluación de programas de medicina sean más explícitos los criterios de evaluación cualitativa.

\section{DISCUSIÓN}

La producción científica de las escuelas de medicina en el Perú se ha incrementado en los últimos años, por ejemplo, de sólo haber dos universidades con más de 13 artículos originales en 10 años para el periodo 2000-2009 en WoS ${ }^{(40)}$, para el periodo $2010-2018$ existen más de 15 universidades que superan ese número. $Y$ como se ha podido apreciar en la figura 1, este incremento coincide con la vigencia de la ley universitaria y el proceso de licenciamiento de universidades, en adición de otras políticas de incentivo a la investigación como el reconocimiento de investigadores REGINA por parte de Concytec. Además de estos cambios estructurales en las políticas de incentivo a la investigación universitaria, varias iniciativas recientes se han desarrollado en las escuelas de medicina, como la implementación de la tesis formato artículo científico ${ }^{(41)}$, profundización del trabajo de las sociedades científicas ${ }^{(42,43)}$ y talleres de formación en publicación científica para estudiantes de medicina.

El proceso de licenciamiento de escuelas de medicina podría generar un mayor impulso a la investigación en las mismas, por lo que se requiere identificar y sistematizar las buenas prácticas de las universidades que son altamente productivas. Así mismo, es importante el trabajo en 
colaboración generando redes no sólo con universidades e instituciones extranjeras, sino también al interior del país y en las mismas ciudades, donde puedan sumar sus capacidades instaladas y potenciar la capacidad nacional de producir investigación de calidad.

Dado que existe un escenario con incentivos de desarrollo académico, profesional o económicos vinculados con la publicación ${ }^{(44)}$ se deben reforzar las actividades de formación en ética para evitar malas prácticas ${ }^{(45,46)}$, más aún en un contexto de proliferación de revistas predadoras ${ }^{(47,48)}$. Por lo que se hace necesario también estimular la implementación de oficinas o comités de integridad científica ${ }^{(49,50)}$.

El proceso de licenciamiento es necesario; sin embargo, hay algunos detalles que aún faltan ser revisados, por ejemplo, la articulación del proceso de licenciamiento de SUNEDU con la acreditación que maneja el Sistema Nacional de Evaluación, Acreditación y Certificación de la Calidad Educativa - SINEACE, pues no se tiene claro si una escuela de medicina ya acreditada debe pasar por el proceso de licenciamiento, o peor aún, que una de estas no sea licenciada por no cumplir las condiciones básicas de calidad.

La articulación entre las instituciones vinculadas con la formación de médicos en el Perú (Universidades, ASPEFAM, CMP y Ministerio de Salud), del sistema de garantía de la calidad universitaria (Ministerio de Educación, SINEACE y SUNEDU) y con la investigación en salud (CONCYTEC y el Instituto Nacional de Salud), es necesaria para mejorar la calidad de los médicos egresados de universidades peruanas. El proceso de licenciamiento de programas de medicina será un hito en la historia de las escuelas de medicina del Perú.

La presencia de indicadores de investigación en el proceso de licenciamiento de programas de medicina será un fuerte estímulo que ayudará a mejorar la investigación en salud en el país, con un consiguiente incremento en la producción científica como se apreció con el licenciamiento institucional. Por ello, es importante que tanto Concytec, como el Instituto Nacional de Salud (entes rectores en investigación a nivel global y en salud, respectivamente), tengan presente este proceso, para que la necesidad de investigar en las universidades debido al licenciamiento pueda vincularse con las prioridades nacionales y regionales de investigación en salud.

Finalmente, consideramos que incluir la medición de indicadores de investigación para la determinación del tiempo de licenciamiento de programas de medicina es un acierto, así como el usar el índice $\mathrm{H}$ sobre el impacto normalizado, y que por el momento para calcular una distribución de quintiles de universidades latinoamericanas es mejor usar el WoS Incites, pero de usar quintiles en un ranking exclusivo de universidades peruanas la mejor opción sería Scopus.

\section{Fuente de financiamiento: autofinanciado}

Conflictos de interés: PMT trabaja actualmente en UCSUR, ha ejercido docencia en UPAO, UPC, UNMSM y UPCH, y ha estudiado en UNMSM, UPAO y UPCH. CJTH ha ejercido docencia en UDCH y ha estudiado en USMP y UPCH. JPM y JAQ han estudiado en UNMSM y JPM ha ejercido docencia en UCV y UPeU.

\section{REFERENCIAS BIBLIOGRÁFICAS}

1. Perú, Congreso de la República. Ley $\mathrm{N}^{\circ}$ 30220: Ley Universitaria. Lima: Congreso de la República; 2014.

2. Jacobs DS, Cross PC. The value of medical student research: the experience at Stanford University School of Medicine. Med Educ. 1995;29(5):342-6. doi: https:// doi.org/10.1111/j.1365-2923.1995. tb00023. $x$

3. Chang Y, Ramnanan CJ. A review of literatura on medical students and scholarly research: experiences, attitudes, and outcomes. Acad Med. 2015;90(8):116273. doi: https://doi.org/10.1097/ ACM.0000000000000702

4. Bonilla-Velez J, Small M, Urrutia R, Lomberk G. The enduring value of research in medical education. Int J Med Students. 2017;5(1):37-44.

5. Taype-Rondan A, Huaccho-Rojas J, Pereyra-Elias R, Mejia CR, MaytaTristan P. Características de los cursos de investigación de las escuelas de medicina del Perú. Arch Med. 2015;11(2):e1.

6. Pereyra-Elías R, Huaccho-Rojas J, TaypeRondán A, Mejía CR, Mayta-Tristan P. Publicación y factores asociados en docentes universitarios de investigación científica en escuelas de medicina del Perú. Rev Peru Med Exp Salud Publica. 2014;31(3):424-30. doi: https://doi. org/10.17843/rpmesp.2014.313.76

7. Atamari-Anahui N, Sucasaca-Rodriguez C, Marroquin-Santa Cruz JA. Publicación científica de asesores de tesis de pregrado en una escuela de medicina de Cusco, Perú. Invest Educ Med. 2016;5(20):27980. doi: https://doi.org/10.1016/j. riem.2016.05.002

8. Alarcon-Ruiz CA, Quezada MA. Publicación de artículos científicos por asesores de tesis de una facultad de medicina. Rev Med Hered. 2018;29(3):152-7. doi:https://doi.org/10.20453/rmh. v29i3.3403.
9. Herrera-Añazco P, Valenzuela-Rodríguez G. Producción científica de los vicerrectores de investigación en universidades peruanas que tienen una facultad de medicina. Medwave. 2017;17(8):e7074.

10. Mayta-Tristán P, Cartagena-Klein R, Pereyra-Elías R, Portillo A, Rodríguez-Morales AJ. Apreciación de estudiantes de Medicina latinoamericanos sobre la capacitación universitaria en investigación científica. Rev med Chile. 2013;141(6):716-22. doi: https://doi.org/10.4067/S003498872013000600005

11. Arroyo-Hernández $\mathrm{CH}$, De la Cruz W, Miranda-Soberon UE. Dificultades para el desarrollo de investigaciones en pregrado en una universidad pública de provincia, Perú. Rev Peru Med Exp Salud Publica. 2008;25(4):448.

12. Huamani C, Dulanto-Pizzorni A, Rojas-Revoredo V. "Copiar y pegar” en investigaciones en pregrado: haciendo 
mal uso del Internet. An Fac Med (Lima). 2008;69(2):117-9. doi: https:// doi.org/10.15381/anales.v69i2.1154

13. Mejia CR, Inga-Berrospi F, Mayta-Tristán P. Titulación por tesis en escuelas de medicina de Lima, 2011: características, motivaciones y percepciones. Rev Peru Med Exp Salud Publica. 2014;31(3):50914. doi: https://doi.org/10.17843/ rpmesp.2014.313.88

14. Mejia CR, Cáceres OJ, Vera CA, IngaBerrospi F, Mayta-Tristán P. Percepción y factores asociados a insatisfacción que los médicos recién graduados tienen de sus asesores de tesis, Lima-Perú. Educ Med Super. 2016; 30(4):340-8.

15. Saldaña-Gastulo JJC, Quezada-Osoria CC, Peña-Oscuvilca A, Mayta-Tristán P. Alta frecuencia de plagio en tesis de medicina de una universidad pública peruana. Rev Peru Med Exp Salud Publica. 2010;27(1):63-7. doi: https://doi.org/10.1590/S172646342010000100011

16. Toro-Huamanchumo CJ, Arce-Villalobos LR, Gonzalez-Martinez J, Melgarejo-Castillo A, Taype-Rondan A. Financiamiento de la investigación en pregrado en las facultades de medicina peruanas. Gac Sanit. 2017;31(6):541-2. doi: https://doi. org/10.1016/j.gaceta.2016.06.009

17. Carvajal-Tapia AE, Carvajal-Rodríguez E. Status of scientific production in medicine in South America. 1996-2016. Rev Fac Med. 2018;66(4):595-600. doi: https://doi.org/10.15446/revfacmed. v66n 4.67215

18. Superintendencia Nacional de Educación Superior Universitaria. El modelo de licenciamiento y su implementación en el sistema universitario peruano. Lima: SUNEDU; 2015.

19. Lavalle C, De Nicolas VL. Peru and its new challenge in higher education: towards a research university. Plos One. 2017;12(8):e0182631. DOI: 10.1371/ journal.pone.0182631

20. Scimago. Ranking Scimago Iberomaérica 2015. Granada: Scimago; 2015.

21. Mayta-Tristán P, Cuentas M, NúñezVergara M. Responsabilidad de las instituciones ante la proliferación de escuelas de medicina en el Perú. Acta Med Peru. 2016;33(3):178-82.

22. Superintendencia Nacional de Educación Superior Universitaria. Modelo de licenciamiento de programa de pregrado de medicina. Lima: SUNEDU; 2018.

23. Superintendencia Nacional de Educación Superior Universitaria. Resolución CD SUNEDU 164-2018: Proyecto de reglamento del procedimiento de licenciamiento del programa de pregrado de medicina. Lima: SUNEDU; 2018.

24. Bornmann L, De Moya F, Mutz R. Do universities or research institutions with a specifc subject profile have an advantage or disadvantage in institutional rankings? A latent class analysis with data from the SCImago Ranking. J Am Soc Inform Sci Technol. 2013;64(11):2310-6. doi: https://doi.org/10.1002/asi.22923

25. De-Moya-Anegón F Herrán-Páez E, Bustos-González A, Corera-Álvarez E, Tibaná-Herrera G. Ranking Iberoamericano de instituciones de educación superior. SIR Iber 2018. Barcelona, España: Ediciones Profesionales de la Información; 2018.

26. Wang Q, Waltman L. Large-scale analysis of the accuracy of the journal classification sytems of Web of Science and Scopus. J Informetrics. 2016;10(2):347-64. doi: https://doi.org/10.1016/j.joi.2016.02.003

27. FranceschiniF,MaisanoD, Mastrogiacomo L. Empirical analysis and classification of databes errors in Scopus and Web of Science. J Informetrics. 2016;10(4):93353. doi: https://doi.org/10.1016/j. joi.2016.07.003

28. Superintendencia Nacional de Educación Superior Universitaria. Informe bienal sobre la realidad universitaria peruana. Lima: SUNEDU; 2018.

29. Sypsa V, Hatzakis A. Assesing the impact of biomedical research in academic institutions of disparate sizes. BMC Med Res Methodol. 2009:9:33. doi: https:// doi.org/10.1186/1471-2288-9-33

30. Vieira ES, Gomes JAN. A research impact indicator for institutions. J Informetrics. 2010;4(4):581-90. doi: https://doi. org/10.1016/j.joi.2010.06.006

31. Bornmann L, Marx W. Methods ofr the generation of normalized citation impact scores in bibliometrics: which method best reflects the judgements of experts? J Informetrics. 2015;9(2):408-18. doi: https://doi.org/10.1016/j.joi.2015.01.006

32. Glanzel W. On the h-index- a mathematical approach to a new measure of publication activity and citation impact. Scientometrics. 2006;67(2):315-21. doi: https://doi.org/10.1007/s11192-0060102-4

33. Molinari JF, Molinari A. A new methodology for ranking scientific institutions. Scientometrics. 2008;75(1):163-74. doi: https://doi.org/10.1007/s11192-0071853-2

34. Torres-Noriega J. Los exámenes nacionales de medicina (ENAM) en el
Perú. Rev Peru Med Exp Salud Publica. 2008;25(3):316-8.

35. Mayta-Tristán P, Poterico JA, GalánRodas E, Raa-Ortiz D. El requisito obligatorio del servicio social en salud del Perú: discriminatorio e inconstitucional. Rev Peru Med Exp Salud Publica. 2014;31(4):781-7. doi: https://doi. org/10.17843/rpmesp.2014.314.134

36. Perú, Ministerio de Salud. RM785-2016/ MINSA: Modifican el Reglamento de la Ley N²3330 Ley del Servicio Rural y Urbano Marginal de Salud-SERUMS. Lima: Ministerio de Salud; 2016.

37. Colegio Médico del Perú [Nota de Prensa]. Egresados de medicina deben aprobar examen nacional de medicina para colegiarse y ejercer la profesión. Lima: CMP; 2019. [Fecha de acceso 13-2-19] Disponible en: https://www.cmp.org.pe/ egresados-de-medicina-deben-aprobarexamen-nacional-para-colegiarse-y-ejercerla-profesion/

38. Varela-Contreras OA, ValladaresGarrido MJ, Toro-Huamanchumo CJ, Taype-Rondan A. Enseñando con las luces apagadas: necesidad de encuestas y evaluaciones a estudiantes de medicina y médicos en Perú. Rev Med Chile. 2017;45(5):680-1. doi: https://doi. org/10.4067/S0034-98872017000500020

39. McDonald G. Does size matter? The impact of student-staff ratios. J Higher Educ Policy Management. 2013;35(6):652-67. doi: https://doi.org/ 10.1080/1360080X.2013.844668

40. Huamaní C, Mayta-Tristán P. Producción científica peruana en medicina y redes de colaboración, análisis del Science Citation Index 2000-2009. Rev Peru Med Exp Salud Publica. 2010;27(3):315-25. doi: https:// doi.org/10.17843/rpmesp.2010.273.1488

41. Mayta-Tristán P. Tesis en formato de artículo científico: una oportunidad para incrementar la producción científica universitaria. Acta Med Peru. 2016;33(2):95-8.

42. Toro-Huamanchumo CJ, Failoc-Rojas VE, Díaz-Vélez C. Participación en sociedades científicas estudiantiles y en cursos extracurriculares de investigación, asociados a la producción científica de estudiantes de medicina humana: estudio preliminar. FEM: Revista de la Fundación Educación Médica. 2015;18(4):293-8. doi: https:// doi.org/10.33588/fem.184.794

43. Taype-RondánÁ,Lajo-Aurazo Y,GutiérrezBrown R, Zamalloa-Masías N, SaldañaGonzales M. Aporte de las sociedades estudiantiles en la publicación científica en SciELO-Perú, 2009-2010. Rev Peru 
Med Exp Salud Publica. 2011;28(4):6912. doi: https://doi.org/10.1590/S172646342011000400022

44. Nieto-Gutierrez W, Fernández-Chinguel JE, Taype-Rondan A, Pacheco-Mendoza J, Mayta-Tristán P. Incentivos por publicación científica en universidades peruanas que cuentan con escuelas de medicina, 2017. Rev Peru Med Exp Salud Publica. 2018; 35(2):353-4. doi: https:// doi.org/10.17843/rpmesp.2018.352.3327

45. Edwards MA, Roy S. Academic research in the 21st century: maintaining scientific integrity in a climate of perverse incentives and hypercompetition. Environ Eng Sci. 2017;34(1):51-61. doi: https://doi. org/10.1089/ees.2016.0223
46. Boutron I, Ravaud P. Misrepresentation and distortion of research in biomedical literature. PNAS. 2018;115(11):2613-9. doi: https://doi.org/10.1073/pnas.1710755115

47. Beall J. Predatory journals: ban predators from the scientific record. Nature. 2016;534(7607):326. doi: https://doi. org/10.1038/534326a

48. Shamseer L, Moher D, Maduekwe O, Tumer L, Barbour V, Clark J, et al. Potential predatory and legitimate biomedical journals: can you tell the difference? A cross-sectional comparison. BMC Med. 2017;15(1):28. doi: https://doi. org/10.1186/s12916-017-0785-9

49. Universidad Científica del Sur. Reglamento del Comité de Integridad Científica. Lima: Universidad Científica del Sur; 2017
50. Forsberg EM, Anthun FO, Bailey S, Birchley G, Bout H, Casonato C, et al. Working with research integrity-guidance for research performing organizations: The Bonn PRINTEGER Statement. Sci Eng Ethics. 2018;24(4):1023-34. doi: https:// doi.org/10.1007/s11948-018-0034-4

Correspondencia: Percy Mayta-Tristán

Dirección: Av. Brasil 2169 Dpto. 802, Jesús

María, Lima, Perú

Teléfono: 51987532133

Correo electrónico:pmayta@cientifica.edu.pe
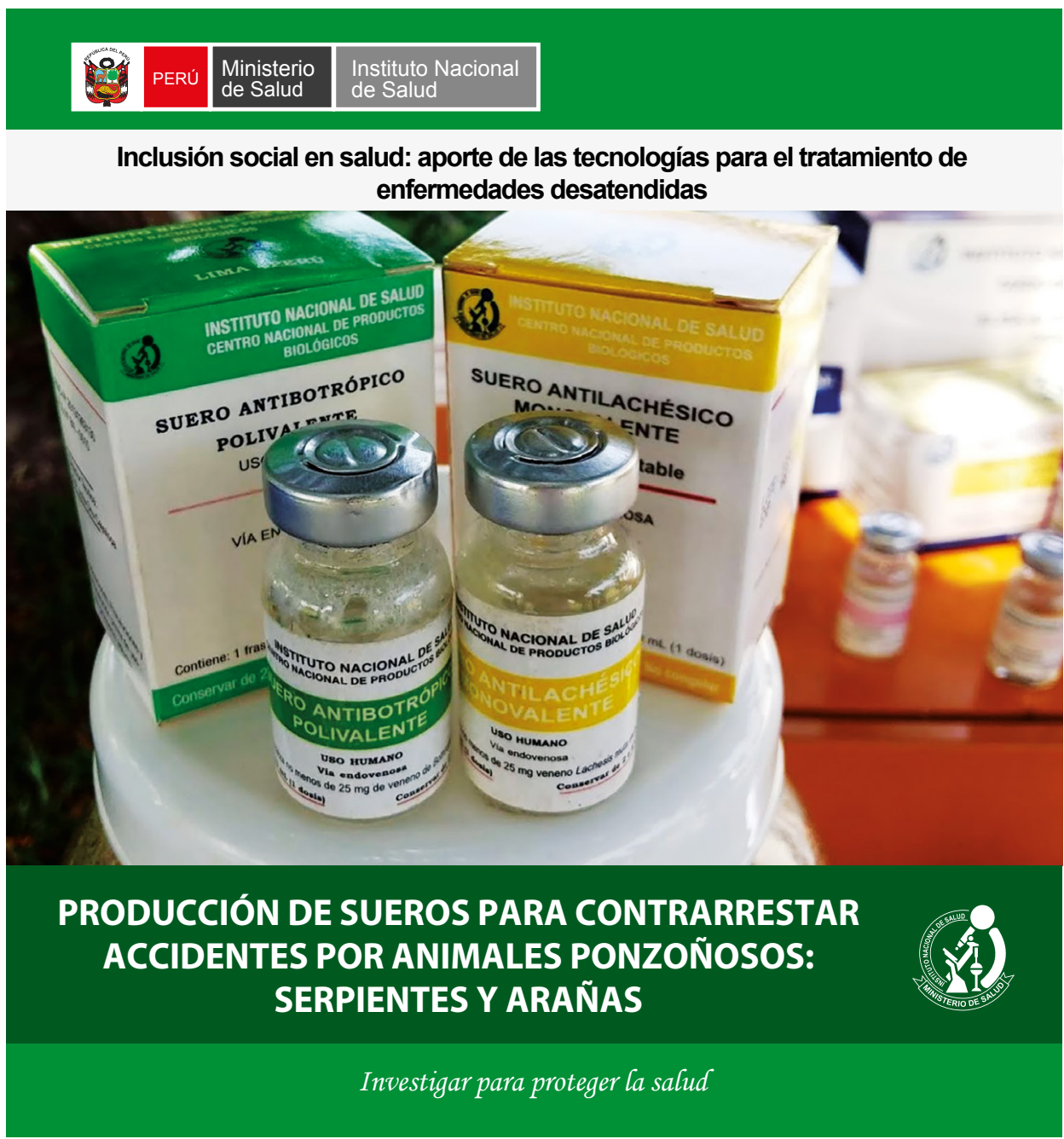